Rhinitis, Rhinosinusitis, Bronchitis - der Übergang ist fließend

\title{
Atemwege als Ganzes betrachten
}

Kommen Patienten mit akutem Husten oder klassischen Rhinosinusitis-Beschwerden in die Praxis, ist es wichtig, die Atemwege als Ganzes zu betrachten und zu therapieren. Darüber waren sich die Mitglieder des neu gegründeten „Kompetenz-Kolleg United Airways“, bestehend aus Experten der HNO-Medizin, der Pneumologie, der Allgemeinmedizin und der Pharmazie, einig. Denn die oberen und unteren Atemwege sind eine anatomische und funktionelle Einheit. Diese Verbindung erklärt, warum es nicht selten zum Etagenwechsel der Erkrankung kommt.

Nicht jedes Sekretolytikum eignet sich jedoch für die ganzheitliche Behandlung von Atemwegsinfekten. Das pflanzliche Spezialdestillat ELOM-080 (GeloMyrtol $^{\circledR}$ forte) ist derzeit das einzige Phytotherapeutikum, das zur Behandlung von akuten und chronischen Bronchitiden und Sinusitiden zugelas- sen ist, sagten die Experten. Das Phytopharmakon aus rektifiziertem Eukalyptus-, Zitronen-, Süßorangen- und Myrtenöl zeigt mukolytische, sekretolytische sowie sekretomotorische Eigenschaften und verbessert die mukoziliäre Clearance (MCC). So dokumentierte eine pharmakokinetische Studie nach Einnahme von ELOM-080 einen deutlichen Anstieg der Sekretausscheidung. Unter Zuhilfenahme der Hochgeschwindigkeits-Videomikroskopie konnte überdies optisch verifiziert werden, dass der Extrakt die Zilienschlagfrequenz des respiratorischen Epithels konzentrationsabhängig signifikant steigert.

Die ätherischen Öle gelangen durch die Blutbahn in die Atemwege, und der Anwender kann bereits nach der Einnahme der ersten Kapsel (mit einem Glas Wasser, eine halbe Stunde vor einer Mahlzeit) freier durchatmen. Die Kapseln lösen sich gezielt im Dünndarm auf,

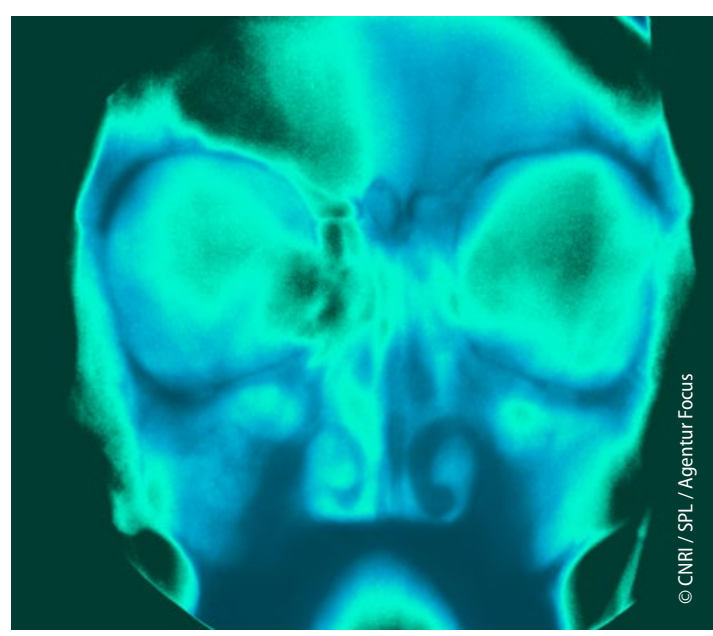

Die akute Sinusitis ist oft nur der Vorbote einer Bronchitis.

wo ELOM-080 nahezu vollständig resorbiert und zu den Atemwegen transportiert wird, um seine Wirkung zu entfalten.

Julia Pflegel

- Journalisten Workshop "GeloMyrtol ${ }^{\circledR}$ forte: Luft für die United Airways"; Hamburg, September 2016 (Veranstalter: Pohl-Boskamp)

\section{Versorgungsrealitåt bei phlebologischen Erkrankungen}

\section{Suboptimaler Einsatz von Kompressionsstrümpfen}

- Die Versorgung von phlebologischen Erkrankungen mit medizinischen Kompressionsstrümpfen (MKS) ist verbesserungsfähig, fasste Dr. Christine Schwahn-Schreiber, Otterndorf, die Ergebnisse der mediven ${ }^{\circledast}$-Beobachtungsstudie zusammen [Schwahn-Schreiber C et al. Phlebologie. 2016;45: 207-14]. Patienten erhalten unabhängig von ihrer Erkrankung und ihrem Gewicht fast immer die gleichen Strümpfe.

\section{Individuellere Versorgung notwendig} 74\% der Patienten erhielten Strümpfe leichter Qualität, 24\% eine mittlere Qualität, aber nur $2 \%$ eine starke Qualität. Noch uniformer ging es hinsichtlich der Kompressionsklassen zu. 98\% wurden mit Kompressionsklasse 2 versorgt. 34\% der Patienten hatten Übergewicht und 28\% eine manifeste Adipositas. Letztere mussten aber dennoch zu zwei Drittel $(64,4 \%)$ mit einer - inadäquaten - leichten Qualität vorliebnehmen.

Auch die CEAP-Klasse, eine Einstufung der Krankheitsschwere, hatte nur einen geringen Einfluss auf die Versorgung der Patienten. Umgekehrt hatten aber die MKS-Qualitäten einen Einfluss auf die Verbesserung der CEAP-Klasse, gibt die Phlebologin zu bedenken. So profitierten C3-Patienten, also mit gravierender Krankheitsausprägung, erheblich mehr von Strümpfen mittlerer Qualität als von solchen leichter Qualität. „Daher wäre eine individuellere Versorgung für die Patienten wünschenswert.“

Reimund Freye

- Satellitensymposium „Versorgungsforschung Phlebologie“, 58. Jahrestagung der Deutschen Gesellschaft für Phlebologie, 17. Dreiländertagung der Deutschen, Österreichischen und Schweizerischen Gesellschaften für Angiologie; Dresden, September 2016 (Veranstalter: medi)

\section{Kurz notiert}

Chronische Hepatitis $\mathrm{C} \rightarrow$ Die Fixkombination Elbasvir $50 \mathrm{mg} /$ Grazoprevir $100 \mathrm{mg}$ (Zepatier $^{\circledast}$ ) zur Behandlung erwachsener Patienten mit einer chronischen Hepatitis-C-Infektion der Genotypen (GT) 1 oder 4 ist ab sofort in Deutschland verfügbar (PZN 11320392). Bei einmal täglicher Einnahme ohne Ribavirin waren bei diesen Patienten hohe Heilungsraten erreicht worden.

Basis für die Zulassung waren Ergebnisse aus acht klinischen Studien, in die etwa $2.000 \mathrm{~Pa}$ tienten mit chronischer Hepatitis-C-Virus(HCV)Infektion eingeschlossen wurden, darunter auch Patienten unter Opioid-Agonisten-Therapie, Patienten mit chronischer Niereninsuffizienz und Patienten mit HIV-Koinfektion. Den primären Endpunkt (SVR 12) erreichten unter einer zwölfwöchigen Therapie insgesamt 93\% der Patienten mit einer Infektion mit dem GT 1a, 96\% der Patienten mit dem GT 1b und 94\% der Patienten mit dem GT 4.

Red.

- Nach Informationen von MSD 